\title{
Aprendizaje y participación del adulto mayor en contextos universitarios
}

\section{Adult people learning and participation in university contexts}

\author{
María Eugenia Narváez Betancur*
}

\begin{abstract}
Resumen
En el presente artículo se evidencia la necesidad de establecer programas formativos dirigidos a la población adulta mayor, con el propósito de implementar propuestas educativas que favorezcan el desarrollo individual y permitan participar adecuadamente en los proyectos de inclusión social, con un carácter integrador y un factor de crecimiento en la vejez, que facilite vislumbrar la educación permanente como un escenario de aprendizaje a lo largo de la vida, a fin de proporcionar una prolongada existencia productiva.
\end{abstract}

Palabras clave: Envejecimiento; Aprendizaje; Educación; Participación.

Tecnóloga en Gerontología, Tecnológico de Antioquia, cortipersianas@yahoo.com 
- Hea María Engenia Narváez Betancur

\section{Abstract}

This paper assumes the necessity to establish formative programs for adult people. It has as a prime purpose to implement educative purposes which will implement the way they can participate in those projects of social inclusion. These factors will let them a continuous learning process through their life time so they will have a continuous productive adult life.

Keywords: Aging; Learning; Education; Participation.

\section{Introducción}

La presencia cada vez más evidente en Colombia de un alto número de personas adultas mayores, muestra un nuevo fenómeno, no solo demográfico, sino, fundamentalmente, en el contexto de lo político, lo social y lo cultural. Apenas una década atrás el envejecimiento de la población era visto como una realidad lejana y propia sólo de los países desarrollados, producto de sus muy bajas tasas de fecundidad y sus altas esperanzas de vida, que sobrepasan las expectativas de una población con mayor longevidad, gracias al mejoramiento de las condiciones dadas por los avances científicos, tecnológicos, médicos, educativos y sociales.

Retomando el artículo del semillero Proyección Gerontológica, de la Facultad de Educación y Ciencias Sociales, de la Institución Universitaria Tecnológico de Antioquia, según Lucy Wartenberg, (2011) representante auxiliar del Fondo de Población de las Naciones Unidas, en el que se promulga que "el mayor reto no es lograr este aumento de la esperanza de vida en la población Adulta Mayor, sino que esta población llegue a una vejez digna”. Vejez digna, precisamente por la pretensión sustantiva de ser participativa, activa y productiva, donde se alineen propuestas de educación permanente e inclusión social.
En el contexto de esta realidad, es necesario hablar del envejecimiento, entendido como aquel camino que comienza a temprana edad en la planificación de una futura vejez con calidad de vida. El proceso de envejecimiento se fusiona con otros aspectos de la maduración que vivencia todo ser humano en las etapas del ciclo vital: infancia, adolescencia, jwentud, adultery vejer:

El envejecimiento se evidencia de manera diferente, con características propias de cada individuo; es innegable el declive que se manifiesta, pero, en nuestra sociedad, percibimos el envejecimiento como un sinónimo de pérdidas o deterioros y no se reconoce que la vejez puede ser un tiempo propicio para la construcción y potencialización de capacidades emocionales, intelectuales y creadoras que son inherentes al ser humano.

Es de vital trascendencia adquirir conocimientos sobre las etapas del envejecimiento porque ello contribuirá a mejorar la percepción y las actitudes hacia la vejez, como lo consideran Dulcey \& Ardila (1976), los esfuerzos médicos y científicos por prolongar la vida no tendrían sentido por sí solos, si no nos esforzamos por mejorar las actitudes hacia esa vida que se prolonga, esto, a través de la educación en todas las generaciones. $\mathrm{Al}$ respecto, Ramón \& Cajal (2008) han escrito que lo que debe preocuparnos no son las arrugas del rostro sino las del cerebro y, añaden, "se habla de envejecer cuando se pierde la curiosidad 
intelectual y cuando, junto a la torpeza de las piernas, se advierte la torpeza de la palabra y del pensamiento".

La importancia de reconocer que el envejecimiento es de carácter universal, progresivo, continuo e irreversible y que cada ser humano es proclive a ello y lo enfrenta a nivel biosicosocial, tiene sus raíces en la idea de que éste puede llevarse a cabo en adecuadas condiciones para alcanzar un envejecimiento exitoso, o, por otra parte, se puede presentar en situaciones no muy adecuadas, lo que implica un envejecimiento patológico. Es decir, el impacto negativo se manifiesta en el cúmulo de pérdidas sicosociales que acontecen durante la vejez y que afecta directamente el estado mental y su potencial funcional, lo que produce una acelerada evolución en las enfermedades. Estos factores parecen determinar, en algunas personas mayores, su incapacidad para percibir sus competencias y habilidades y se manifiesta una auto insatisfacción o baja autoestima, la cual se puede considerar el eje de la calidad de vida de las personas mayores, puesto que es uno de los pilares que sustentan el desarrollo de la personalidad en todas sus esferas y dimensiones.

Sin duda, un envejecimiento exitoso permite alcanzar una buena salud, tanto física como mental e, incluso, la longevidad de las personas que puede asociarse con el buen desempeño de roles sociales, como; la relación con la pareja implicando estabilidad emocional y afectiva. La relación con los hijos, es uno de los andamiajes que constituye vínculos fuertes, puesto que se presenta la inversión de roles "cuidar y ser cuidados”. La relación con los nietos; los abuelos, como fuente de amor y apoyo, desempeñan roles como: padre sustituto, abuelo divertido, figura distante, depositario de sabiduría familiar. La relación con los amigos, entre otros.
Respecto al buen desempeño de los roles sociales, se rescata la coyuntura de compartir con otras personas actividades de estimulación cognoscitiva, ayudar a controlar tensiones $\mathrm{y}$ presiones de la vida cotidiana. Además, se presenta la tendencia a fortalecer los vínculos con las viejas amistades, lo que quiere decir que una actitud optimista frente a la vida, una autoimagen positiva y la conservación de la autonomía, son características vitales para un envejecimiento exitoso, si se tiene como precedente el encuentro con el otro.

Por consiguiente, se deben tener presente los factores que se manifiestan en las últimas etapas del ciclo vital, así como los cambios de tipo sicosocial. Estos son: las formas de convivencia, las relaciones sociales y la etapa de la jubilación; en lo cognitivo: las alteraciones de las funciones superiores. Éstas son: atención, memoria, inteligencia, praxis y lenguaje; $y$, en lo que tiene que ver con lo afectivo: se da en los cambios de roles, las relaciones familiares y la sociabilidad se ven disminuidas, pues, en ocasiones, los grupos se ven minimizados por las diferencias generacionales.

En procura de promover un envejecimiento activo y exitoso, surge la creación de propuestas de programas académicos para adultos mayores que proporcionan al individuo herramientas para conservar su autonomía y le brindan los medios para alcanzar un mejor equilibrio entre su progreso personal, sus relaciones familiares y sociales y los ambientes de aprendizaje a lo largo de la vida, como ejercicio de una prolongada existencia productiva.

Los programas académicos buscan implementar un método que estimule la creatividad, la necesidad de explorar nuevos conocimientos y favorece la acción participativa-transformadora 
de la realidad del educando. Para tal efecto, se parte del reconocimiento de la misma y busca despertar en el educando una capacidad de razonamiento e interpretación de su realidad para facilitar herramientas que le permitan asumir posiciones críticas y que lo incitan a generar cambios para transformar su entorno, como tendencia que origina la socialización para el cambio y no la simple adaptación. Se aprecia el auge de las corrientes innovadoras de la Pedagogía del adulto, basadas en la participación y uno de los exponentes es el pedagogo brasileño Paulo Freire.

Para dar respuesta a esta necesidad, la Institución Universitaria Tecnológico de Antioquia, adelanta un proyecto piloto de implementación de una universidad para adultos mayores, como propuesta educativa hacia la consolidación de espacios académicos que conciban la educación para toda la vida.

\section{Metodología}

La propuesta está orientada por el método de la investigación cualitativa y la metodología de la investigación acción participativa, cuya finalidad es aportar en la resolución de problemas de una población con sus recursos y participación. Además, busca generar un discernimiento liberador a partir del propio conocimiento popular, que va estructurándose mediante la investigación hecha por la propia población y en la que los investigadores son facilitadores de instrumentos metodológicos que dan lugar a un empoderamiento o consolidación de estrategias de acción para el cambio. Como estrategia metodológica se han vinculado conocimientos, empoderamientos y acciones locales con otras investigaciones similares en otros lugares, con el objeto de vislumbrar homólogos en la construcción de programas educativos que permitan analizar variables administrativas, pedagógicas y de personas mayores de la institución de educación superior. Por tal razón, se emprenden mecanismos como la indagación a expertos y población adulta mayor para ampliar la mirada y poder transformar realidades sociales.

Los módulos propuestos en el proyecto de investigación, materializado y llevado a cabo en la realización del diplomado Educación permanente e inclusiva para los adultos mayores, como prueba piloto en el Tecnológico de Antioquia, corresponden a: Gerontología, Sistemas, Literatura, Mantenimiento cognitivo, Gimnasia oriental e Historia y cultura.

La población participante en la prueba piloto es de 40 personas, con las siguientes características: 31 el $77.5 \%$ mujeres y 9 el $22.5 \%$ hombres; sus edades oscilan entre: 50 55 años, el 12\%; de 56-60 años, el 25\%; de 6165 años, el 32.5\%; de 66-70 años, el 23\%; de 71-75 años, el 7.5 \%; estado civil, 2 de ellos el $5 \%$ solteros, 5 de ellos el $12.5 \%$ viudos, 8 de ellos el 20\% separados y 25 de ellos el $62.5 \%$ casados. Se caracteriza por ser un grupo heterogéneo y su diversidad se ve manifestada en el nivel de formación académica: 6 alumnos el $15 \%$ en nivel bajo-primaria, 17 alumnos el $42.5 \%$ nivel medio-bachiller, 7 alumnos el $17.5 \%$ nivel medio superior, 10 alumnos el 25\% nivel superior-profesional, nivel socioeconómico: estrato 3, 29 de ellos el $72.5 \%$, estrato 4,5 de ellos el $12.5 \%$, estrato 5 , 6 de ellos el $15 \%$. 


\section{Resultados}

Durante la ejecución de la prueba piloto se observa que las personas mayores constituyen uno de los grupos más activos y participativos, tanto en lo referido a la inclusión en programas universitarios de extensión académica, comoal desempeño de su actividad para difundirlos y replicarlos en su entorno.

Se percibe que el aprendizaje es más eficaz cuando se da respuesta a las necesidades que siente el adulto mayor en el rol de estudiante; razón por la cual hay manifestaciones positivas en la participación de aquellos que han tenido bajas oportunidades en la formación académica; es preciso considerar que la participación activa y receptiva es imprescindible para que se propicien espacios académicos que contribuyan a enriquecer el modelo de aprendizaje significativo; y su objetivo principal es que el adulto mayor aprenda a atender, analizar y contextualizar los conocimientos nuevos en su entorno, a partir de la experiencia vivida durante su ciclo vital. El papel esencial del aprendizaje es proporcionar elementos que le permitan al individuo trasformar su realidad.

La ejecución del proyecto piloto, evidencia elementos fundamentales para la realización de programas educativos a lo largo de la vida.

El surgimiento de propuestas innovadoras en programas de educación permanente e inclusiva para adultos mayores, es una de las razones que motivan a este grupo poblacional a participar en la implementación del proyecto piloto.

Podría decirse que el aprendizaje fue transversalizado porque se recibieron conocimientos que enriquecieron la formación académica. Por parte de los estudiantes, desmitificando los paradigmas existentes sobre el aprendizaje en esta etapa de la vida, y queda demostrado no solo su grado de compromiso e interés, sino, también, su capacidad de transmitir su experiencia y conocimiento adquiridos durante su ciclo de vida. El contacto directo con los adultos mayores permite identificar sus necesidades, reconocer sus dificultades y disfrutar la superación de retos.

Gracias al acompañamiento de profesionales idóneos para la orientación de cada módulo, evidenciando cambios comportamentales que experimentan desde el punto de vista personal y reflejan tanto en su entorno familiar como en el social, lo que mejora la calidad de las relaciones afectivas $\mathrm{y}$, con el paso del tiempo, encentrándose inmersos en una experiencia innovadora y transformadora de su realidad.

Los elementos que se describen a continuación, son los que evidencian y comparten en los espacios académicos con el grupo de 40 adultos mayores en la implementación del proyecto piloto "UAM, Universidad para Adultos Mayores".

- Participación activa en la realización de las actividades propuestas, haciendo referencia a la motivación y a la disposición que tiene el adulto mayor para adquirir conocimientos nuevos, aplicarlos a realidad y socializarlos en grupo. Se ha convertido en un reto personal desmitificar los estereotipos sobre la vejez, impuestos por la sociedad y demostrando que el aprendizaje se da a lo largo de la vida. Se concibe la vejez como una etapa más del ciclo vital, donde logra ser proactivo y tener una actitud positiva frente a la vida para mejorar la interacción con su entorno.

En el módulo de Gerontología es importante resaltar la participación activa 
en la presentación frente a sus compañeros. La receptividad frente a la adquisición de nuevos conocimientos sobresale en la experiencia, interesante y novedosa, que se observa con la visita guiada a la biblioteca Humberto Saldarriaga Carmona, ubicada en la Institución Universitaria Tecnológico de Antioquía. Allí, se les permitió a los alumnos interactuar en los espacios académicos para que les dieran uso correcto a las herramientas como libros y revistas indexadas.

- Habilidades y destrezas manifiestas en ejecución de las actividades. Brindarle herramientas al adulto mayor para explorar y descubrir las capacidades que tiene para adquirir conocimientos nuevos y asociarlos con sus vivencias, significó que emergieran sentimientos y emociones indescriptibles con palabras, que se hicieron manifiestas en los cambios y transformaciones que han experimentado en sus vidas en las esferas emocionales, espirituales, familiares $\mathrm{y}$ sociales.

En el módulo de Sistemas se manifiestan dos situaciones: la expectativa y ansiedad de los alumnos que por primera vez tienen contacto con la tecnología y el inmenso deseo de adquirir conocimientos nuevos sobre sistemas y redes virtuales, y aquellos alumnos que tienen conocimientos previos, se muestran ansiosos sobre qué más van a aprender y cuánto van a avanzar.

En esta experiencia se devela una mezcla de sensaciones, emociones y expectativas frente a la ejecución de la práctica. Haciendo énfasis en los valores relevantes como; la cooperación y la solidaridad observada entre los estudiantes.
Los estudiantes manifiestan el grado de satisfacción por los logros obtenidos, a su vez, la participación activa del educando, estimulada por el nivel de aprendizaje logrado y los conocimientos básicos alcanzados con respecto al manejo de los medios tecnológicos y el convencimiento de la necesidad latente que tiene este grupo poblacional de conocer esta herramienta para interactuar en su entorno familiar y social.

- Grado de satisfacción en la asignación de las tareas a desarrollar.

Actitud ante las actividades. Se concibe que la actitud es la manera de comportarse ante una situación determinada, es la disposición con la que se afronta la realidad. Para lograr una participación activa en la ejecución de cualquier actividad se requiere una actitud positiva. La experiencia con los adultos mayores en este escenario de aprendizaje, confirma que son receptivos para la asimilación de nuevos conocimientos y saberes; se manifiesta que el nivel de aprendizaje es más efectivo si está basado en la premisa "Aprender- haciendo".

Durante la ejecución del módulo de Literatura, y la realización de actividades académicas, se propician espacios en los que los adultos mayores despiertan la capacidad de expresar la transparencia de sus sentimientos, con la evocación de sus recuerdos y vivencias.

En el transcurso de las clases se ha visto fortalecido el trabajo en equipo, se han consolidado los vínculos de compañerismo $y$ se ha estimulado la creatividad en la construcción de las composiciones 
literarias. Los estudiantes, por iniciativa propia, consultan, indagan y exploran conocimientos nuevos para mejorar la participación.

- Grado de comunicación en el espacio académico (profesor - estudiantes y entre compañeros). Es significativa la presencia de profesionales idóneos orientando estos programas educativos, porque propician espacios de buena comunicación y respeto por la palabra del otro, se establecen diálogos de saberes. La generación de un buen ambiente en el espacio académico estimula una participación activa y tranquila para el estudiante.

- Uso adecuado de la herramienta metodológica utilizada en la formación despierta el interés de los adultos mayores por las temáticas abordadas por el docente y los estimula a indagar más para ampliar los conocimientos.

En la realización de los módulos de Mantenimiento cognitivo y Gimnasia oriental, los estudiantes exploran nuevas técnicas para auto reconocer su humanidad, son personas entusiastas, alegres, dinámicas, abiertas a experimentar sensaciones que se reflejan en cambios en su comportamiento. Con un cine foro de la película "Mas allá de la luz", del filósofo francés René Mey, propiciando un espacio de encuentro intergeneracional, con la participación activa de familiares y estudiantes.

En las actividades de Gimnasia oriental, los estudiantes tomaron conciencia de la importancia de tener una salud óptima, por medio de hábitos saludables y el reconocimiento de su cuerpo le proporciona al adulto mayor el uso pleno de sus facultades y el aprovechamiento de sus potencialidades y habilidades en el campo físico, mental y espiritual, que repercuten en el mejoramiento de sus relaciones afectivas y le permite replicar sus conocimientos $y$ experiencias.

La planeación de salidas de intercambio que se hicieron a: Club de la Edad Dorada, de Comfenalco, Universidad Eafit al grupo "Saberes de vida" y, por último, al Palacio de la Cultura de la ciudad de Medellín. Propuestas innovadoras, degranaceptaciónyparticipación del grupo, la creación de espacios diferentes a los académicos les permitió interactuar en contextos distintos.

Se infiere que para los educandos todas las temáticas orientadas en los diferentes módulos son de gran interés, pero la limitación de tiempo condiciona la adquisición de nuevos conocimientos y se manifiesta la necesidad de profundizar y ampliar las temáticas de cada módulo.

\section{Conclusiones}

- La característica de que el programa académico no sea evaluativo, estimula y no condiciona la participación de los estudiantes.

- La participación es eficaz y transformadora cuando el ambiente de aprendizaje es significativo porque la realidad está contextualizada de acuerdo con las necesidades de cada individuo.

- En los programas educativos dirigidos a adultos mayores, más que aprender nuevos conocimientos es imperante 
propiciar espacios de "encuentro de saberes".

- La actitud positiva frente al proceso de aprendizaje reafirma que son receptivos frente a la asimilación de nuevos conocimientos y saberes. Se manifiesta que el nivel de aprendizaje es más efectivo cuando se basa en el adagio de que es preciso "Aprender-Haciendo".

- La dinámica grupal generada por los docentes causó un impacto positivo sobre la calidad de vida del adulto mayor porque contribuyó en la creación de espacios de interacción y participación como sujetos socializados con una incidencia social y política.

\section{Discusión de resultados}

Es un reto mundial, para todas las universidades, la creación de propuestas educativas que beneficien la población adulta mayor. En la actualidad, en España existen aproximadamente cuarenta universidades que cuentan con la participación de aproximadamente 30.000 personas que hacen parte de programas universitarios para la población adulta mayor.

Los programas de educación para mayores, de educación no formal, buscan conseguir la acreditación y reconocimiento (como educación no formal, se considera más abierta y flexible) para acceder a otros estudios. En procura de constituir una educación permanente, desde las premisas: Aprender a aprender, aprender haciendo y aprender por competencias.

La mayor motivación para el adulto mayor es ingresar a estas actividades con un único fin de realización personal y no de formación académica con fines profesionales y/o laborales, manifestando un interés personal por aprender, además, el deseo de tener contacto con la realidad de la sociedad actual y no quedarse aislados como individuos, para mejorar y potenciar sus relaciones personales y favorecer el desarrollo individual y grupal. Se pretende crear nuevos paradigmas de formación en los que se establezcan conceptos claros de interacción entre envejecimiento proactivo, prevención de la dependencia o promoción de la autonomía y, en especial, una lucha contra la exclusión social por la desvinculación laboral.

En América Latina, las experiencias educativas universitarias para adultos mayores, son propuestas innovadoras que generan un impacto positivo en la población adulta mayor.

En la actualidad, es menester resaltar algunas de las experiencias, como la de la Pontificia Universidad Católica del Perú; el desafío es la construcción de una alternativa educativa dirigida a personas mayores, ofrecida por primera vez en forma masiva desde el aula universitaria, con objetivos que contribuyen a mejorar la calidad de vida del adulto mayor y a promover el progreso personal y la formación educativa.

En Colombia, la Universidad Eafit, a partir de año 2001, implementó programas educativos dirigidos a personas mayores de 55 años con ganas de "disfrutar" la vida universitaria y vivir la academia desde la perspectiva del deleite intelectual. 


\section{Referencias}

Dulcey, E \& Ardilla, R. (1976). Actitudes hacia los ancianos. Revista latinoamericana de Sicología, (8), 57-67.

Hincapié Gil, Nora. (2011). Proyecto piloto de implementación de la universidad para adultos mayores. Tecnológico de Antioquia, Colombia.

López, de Vera. B. (2010). Retos y expectativas de los programas universitarios para adultos mayores. Una revisión a partir de la práctica. Palabras mayores, \# 4. Recuperado de .http://www.pucp.edu. pe/palabras mayores/nodo/122 \#4 Año marzo 2010

Http://www.monografias.com/ trabajos29/aprendizaje-adulto-mayor/ aprendizaje-adulto-mayor.shtml.

Ramón \& Cajal (2008). Diez frases que hacen pensar a los adultos mayores. Recuperado de http://www.demayores. com/2008/08/26/10-frases-que-hacenpensar-a-los-adultos-mayores/

Tecnológico de Antioquia. (2011). Semillero de Gerontología. Factores que determinan que los hombres adultos mayores decidan no participar en procesos grupales comunitarios e institucionales de deporte, recreación y cultura en la ciudad de Medellín. Medellín.

Universidad de Alicante. (2011). IV Congreso Iberoamericano de Universidades para mayores. España. 